\title{
TOPOLOGY FOR GLOBAL AVERAGE CONSENSUS
}

\author{
Soummya Kar and José M. F. Moura \\ Department of Electrical and Computer Engineering \\ Carnegie Mellon University, Pittsburgh, PA 15213 USA \\ (e-mail:\{moura\}@ece.cmu.edu)
}

\begin{abstract}
We consider the problem of global average-consensus in a network of sensors. The communication among sensors is restricted by the underlying network, where each sensor can exchange information only with its neighbors. We analyze the influence of the network topology on the convergence speed of the iterative consensus algorithm and focus on the design of network topology with respect to this optimality criterion. Topology optimization is a difficult combinatorial optimization problem. We reduce it to a spectral graph design problem, namely, maximizing the ratio $\gamma$ of two eigenvalues of the Laplacian matrix $L$ of the graph. We consider a class of expander graphs, called Ramanujan graphs, for which we find a lower bound on $\gamma$. Through numerical studies, we show that the consensus algorithm converges much faster on the Ramanujan graphs than on structured graphs, on Erdös-Renýi random graphs, and on graphs exhibiting small-world properties.
\end{abstract}

\section{INTRODUCTION}

The paper studies the problem of network graph design in large sensor network applications. Specifically, we consider the design of optimal networks (the connectivity pattern among the sensors, i.e, specifying with which sensors should each sensor in the network communicate) for average consensus in large sensor applications. In [1] network consensus algorithms were studied and in [2] distributed consensus was used for detection in sensor networks and the topology optimization problem was addressed. In this paper, we focus on the design of topologies which optimize the convergence speed of distributed average consensus algorithms. We consider the problem of designing the optimal network topology for $N$ sensors, given the total number of communication links $M$ between sensors.

The problem of finding the optimal topology over the space of all possible networks is a very difficult combinatorial optimization problem. We show that for average consensus it

This work was partially supported by the DARPA DSO Advanced Computing and Mathematics Program Integrated Sensing and Processing (ISP) Initiative under ARO grant \# DAAD 19-02-1-0180 and by NSF under grants \# ECS-0225449 and \# CNS-0428404. is equivalent to an algebraic eigenvalue problem, where the optimality is governed by the ratio $\gamma$ of the algebraic connectivity of the graph to the largest eigenvalue $\lambda_{N}(L)$ of the graph Laplacian matrix $L$. The algebraic connectivity of a graph is the second smallest eigenvalue $\lambda_{2}(L)$ of its discrete Laplacian, [3], [4]. We reduce the problem of maximizing convergence speed to the problem of maximizing the ratio $\gamma$. To this end, we consider a class of expander graphs, [5], called Ramanujan graphs. We find for non-bipartite Ramanujan graphs a lower bound on $\gamma$. It follows from results in spectral graph theory literature that this lower bound for the Ramanujan graphs is, in fact, an upper bound for many classes of graphs. Through numerical studies, we show that the Ramanujan graphs are remarkable in terms of convergence speed and perform much better than structured graphs, random ErdösRényi graphs and graphs exhibiting small-world property [6, $7,8]$.

Section 2 contains preliminaries on algebraic graph theory. Section 3 presents the distributed consensus algorithm and section 4 reduces the convergence speed optimization problem to maximizing the ratio $\gamma$ of the Laplacian matrix $L$. Section 5 states some results from algebraic graph theory and establishes a lower bound on the ratio $\gamma$ for Ramanujan graphs. Section 6 describes an explicit construction of Ramanujan graphs [9] and finally section 7 provides numerical results on the performance of Ramanujan graphs and present a comparative study with other topologies. A more detailed discussion of these results are in [10].

\section{SPECTRAL GRAPH THEORY PRELIMINARIES}

The topology of the sensor network is given by the graph $G=$ $(V, E)$, with nodes $v_{i} \in V, i \in \mathcal{I}=\{1, \ldots, N\}$, and edges the unordered pairs $e=\left(v_{i}, v_{j}\right)$, or, simply, $e=(i, j)$. A graph is simple if it has no loops or multiple edges. The graphs in this paper are assumed to be simple connected graphs. To every graph, we can assign an $N \times N$ adjacency matrix $A$ (where $N=|V|)$, defined by,

$$
a_{i, j}= \begin{cases}1 & \text { if }(i, j) \in E \\ 0 & \text { otherwise }\end{cases}
$$


Since the adjacency matrix $A$ is symmetric, all its eigenvalues are real. The set of neighbors of node $i$ is denoted by $\Omega_{i}=\{j:(i, j) \in E\}$. The degree of node $i$ is the number of its neighbors and is denoted by $\operatorname{deg}(i)$. The degree matrix, $D$ is the $N \times N$ diagonal matrix with the $i$-th entry $\operatorname{deg}(i)$.

The Laplacian $L$, [4] of the graph is the $N \times N$ matrix defined by

$$
L=D-A
$$

The Laplacian is a symmetric, positive semi-definite matrix, and, consequently, all its eigenvalues are non-negative. For a connected graph, we can arrange the eigenvalues of the Laplacian $L$ as,

$$
0=\lambda_{1}(L)<\lambda_{2}(L) \leq \ldots \leq \lambda_{N}(L)
$$

see [4]. The eigenvalue $\lambda_{2}(L)$ is called the algebraic connectivity of the graph.

\section{CONSENSUS ALGORITHM}

Consensus Algorithm: The consensus algorithm computes in a distributed fashion the average of $N$ quantities $r_{n}, n=$ $1, \cdots, N$. Assume a sensor network with connectivity graph $G=(V, E)$. Initially, sensors take measurements $r_{1}, \ldots r_{N}$. We compute their mean $\bar{r}=\frac{1}{N} \sum_{n=1}^{N} r_{n}$ in a distributed fashion using the average consensus algorithm according to the following linear operation, [11],

$$
\mathbf{x}(i)=W \mathbf{x}(i-1)
$$

where, $\mathbf{x}(i)=\left[x_{1}(i) \cdots x_{N}(i)\right]^{T}$ denotes the vector of states at iteration $i$ with $\mathbf{x}(0)=\left[r_{1} \cdots r_{N}\right]^{T}$. W is the weight matrix with $W_{n l}$ denoting the weight associated with link $(n, l)$. For $n \neq l$, the weight $W_{n l}=0$ when there is no link associated with it, i.e., if $(n, l) \notin E$. If $\lim _{i \rightarrow \infty} W^{i}=\mathbf{1 1}^{T}$, then convergence occurs, i.e., $\lim _{i \rightarrow \infty} \mathbf{x}(i)=\overline{\mathbf{x}}=\bar{r} \mathbf{1}$, where the $N$-dimensional vector $\mathbf{1}=[1 \cdots 1]^{T}$.

Link Weights: In this paper we consider the case of equal weights, i.e., we assign an equal weight $\alpha$ to each link in the network. From [11], the optimum equal weight for a given topology is given by,

$$
W_{n l}= \begin{cases}\alpha^{*} & \text { if }(n, l) \in E \\ 1-\alpha^{*} \operatorname{deg}(n) & \text { if } n=l \\ 0 & \text { otherwise }\end{cases}
$$

where $\alpha^{*}=\frac{2}{\lambda_{2}(L)+\lambda_{N}(L)}$. The weight matrix is then given by,

$$
W=I-\alpha^{*} L
$$

where $I$ and $L$ are the $N$-dimensional identity matrix and the graph Laplacian.

The eigenvalues of $W$ are given by, $\gamma_{n}=1-\alpha^{*} \lambda_{n}(L)$. From the spectral properties of the Laplacian of a connected graph, and the choice of $\alpha^{*}$, the eigenvalues of $W$ satisfy $1=\gamma_{1}>$ $\gamma_{2} \geq \cdots \geq \gamma_{N}$, with $\forall n>1: \quad 0 \leq\left|\gamma_{n}\right|<1$ and $\forall n>1$ : $\gamma_{2} \geq\left|\gamma_{n}\right|$.

Convergence rate of the consensus algorithm: It can be shown from eqn.(4) that for any connected graph $G$, the convergence rate of the consensus algorithm is

$$
\left\|\mathbf{x}_{i}-\overline{\mathbf{x}}\right\| \leq\left\|\mathbf{x}_{0}-\overline{\mathbf{x}}\right\| \gamma_{2}^{i}
$$

where

$$
\begin{aligned}
& \gamma_{2}=\frac{1-\gamma}{1+\gamma} \\
& \gamma=\frac{\lambda_{2}(L)}{\lambda_{N}(L)}
\end{aligned}
$$

\section{PROBLEM REDUCTION}

From eqn.(7) it follows that, to obtain the optimal convergence rate, $\gamma_{2}$ should be as small as possible. From eqns.(7) and (9) we note that

the minimum value of $\gamma_{2}$ is attained when the ratio $\gamma=$ $\frac{\lambda_{2}(L)}{\lambda_{N}(L)}$ is maximum. We thus restate the convergence speed optimization problem as:

$$
\max _{G \in \mathcal{G}} \gamma=\max _{G \in \mathcal{G}} \frac{\lambda_{2}(L)}{\lambda_{N}(L)}
$$

where $\mathcal{G}$ denotes the set of all possible graphs with $N$ vertices and with $M$ edges.

\section{SPECTRUM OF REGULAR GRAPHS AND RAMANUJAN GRAPHS}

This section presents some results from spectral graph theory for regular connected graphs and establish a lower bound on the ratio $\gamma$ for the class of Ramanujan graphs. A graph is called $k$-regular if all the vertices have the same degree $k$. For connected regular graphs, we can arrange the eigenvalues of the adjacency matrix $A$ as, $k=\lambda_{1}(A)>\lambda_{2}(A) \geq \ldots \geq$ $\lambda_{N}(A) \geq-k$. The integer $-k$ is an eigenvalue of $A$ iff the graph is bipartite, see [12]. Hence, for non-bipartite graphs, $\lambda_{N}(A)>-k$. For $k$-regular graphs, the eigenvalues of $A$ and $L$ are related by

$$
\forall n \in \mathcal{I}: \quad \lambda_{n}(L)=k-\lambda_{n}(A)
$$

We state a well-known result from algebraic graph theory.

Theorem 1 (Alon and Boppana [13]) Let $G=G_{N, k}$ be a $k$ regular graph on $N$ vertices. Denote by $\lambda_{A}(G)$, the absolute value of the largest eigenvalue (in absolute value) of the adjacency matrix $A$, which is distinct from $\pm k$; in other words, $\lambda_{A}^{2}(G)$ is the next to largest eigenvalue of $A^{2}$. Then

$$
\liminf _{n \rightarrow \infty} \lambda_{A}(G) \geq 2 \sqrt{k-1}
$$


Thus, for the case of families of $k$-regular graphs, $2 \sqrt{k-1}$ is an asymptotic lower bound on the value of $\lambda_{2}(A)$ [9].

Definition 2 (Ramanujan Graphs) A graph $G=G_{N, k}$ will be called Ramanujan if

$$
\lambda_{A}(G) \leq 2 \sqrt{k-1}
$$

Graphs with small $\lambda_{A}(G)$ are called expander graphs, and the Ramanujan graphs are one of the best explicit expanders known. Hence, for non-bipartite Ramanujan graphs, $\lambda_{2}(A) \leq$ $2 \sqrt{k-1}$ and $\lambda_{N}(A) \geq-2 \sqrt{k-1}$.

Then from eqn (11) we have for non-bipartite Ramanujan graphs, $\lambda_{2}(L) \geq k-2 \sqrt{k-1}$ and $\lambda_{N}(L) \leq k+2 \sqrt{k-1}$. Hence, for non-bipartite Ramanujan graphs

$$
\gamma_{2}=\frac{\lambda_{2}(L)}{\lambda_{N}(L)} \geq \frac{k-2 \sqrt{k-1}}{k+2 \sqrt{k-1}}
$$

Theorem 1 and eqn. (12) show that, for general graphs, $\lambda_{A}(G)$ is in the limit lower bounded by $2 \sqrt{k-1}$, while for Ramanujan graphs $\lambda_{A}(G)$ is, for every finite $N$, upper bounded by $2 \sqrt{k-1}$. This together with eqn. (14) hints that consensus algorithms on Ramanujan graph topologies should have very good convergence properties.

\section{EXPLICIT CONSTRUCTION OF RAMANUJAN GRAPHS}

We now provide explicit constructions of Ramanujan graphs. The explicit constructions presented next are based on the construction of Cayley graphs. The following paragraph gives a brief overview of the Cayley graph construction.

Cayley Graphs: The Cayley graph construction uses group theory to construct $k$-regular graphs. Let $X$ be a finite group with $|X|=N$, and $S$ a $k$-element subset of $X$, i.e., $s \in S$ implies $s^{-1} \in S$. We now construct a graph $G=G(X, S)$ by having the vertex set to be the elements of $X$, with $(u, v)$ as an edge if and only if $v u^{-1} \in S$. The graph constructed above is $k$-regular on $|X|$ vertices. The subset $S$ is often called the set of generators of the Cayley graph $G$, over the group $X$.

\subsection{LPS: Explicit Constructions of Ramanujan Graphs}

Explicit constructions of Ramanujan graphs for a fixed $k$ and varying $N$, [5], have been described for the cases $k-1$ is a prime, [9], [14], or a prime power, [15]. We describe a nonbipartite Ramanujan graph construction by Lubotzky-PhillipsSarnak [9] and call them LPS-II graphs.

LPS-II Construction [9]: We start with two unequal primes $p$ and $q$ congruent to $1 \bmod 4$, such that the Legendre symbol $\left(\frac{p}{q}\right)=1$. We define the set $P^{1}\left(F_{q}\right)=\{0,1, \ldots, q-$ $1, \infty\}$ (Projective Line over $F_{q}$ ), which is basically the set of

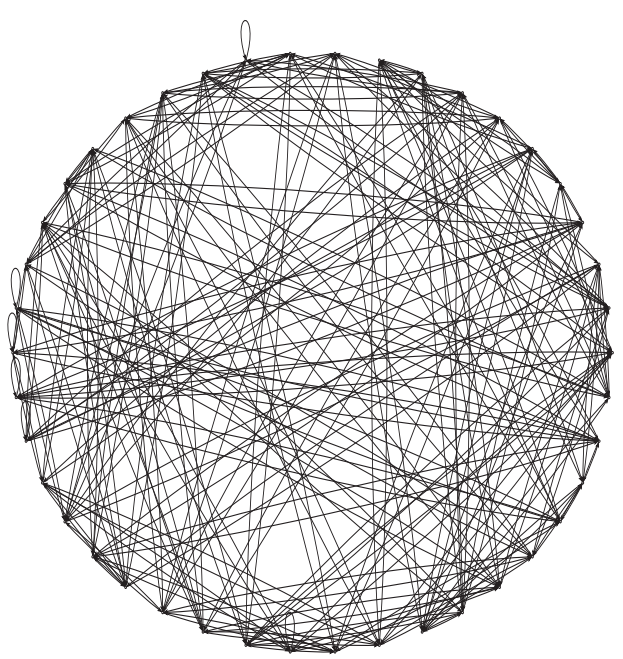

Fig. 1. LPS-II graph with number of vertices $N=42$ and degree $k=6$ (Figure constructed using software Pajek.)

integers modulo $q$, with an additional "infinite" element inserted in it. It follows that $\left|P^{1}\left(F_{q}\right)\right|=q+1$. Let $i$ be an integer satisfying $i^{2} \equiv-1 \bmod (q)$. We consider $p+1$ matrices in PSL(2,Z/qZ)(Projective Special Linear Group) given by,

$$
\widetilde{\beta}=\left(\begin{array}{rr}
a_{0}+i a_{1} & a_{2}+i a_{3} \\
-a_{2}+i a_{3} & a_{0}-i a_{1}
\end{array}\right)
$$

where $\beta=\left(a_{0}, a_{1}, a_{2}, a_{3}\right)$ is a solution of the equation $a_{0}^{2}+$ $a_{1}^{2}+a_{2}^{2}+a_{3}^{2}=p$ such that $a_{0}>0$ and odd, and $a_{j}$ even for $j=1,2,3$ (there are exactly $p+1$ such solutions as given by a formula of Jacobi [16].) These $p+1$ matrices constitute the set $S$ of generators. The LPS-II graphs are produced by the action of the set $S$ on $P^{1}\left(F_{q}\right)$, in a linear fractional way. The Ramanujan graphs obtained in this way, are non-bipartite $p+$ 1-regular graphs on $q+1$ vertices [9]. The LPS-II graphs thus obtained, may few loops [17], which does not pose problems because their removal does not affect the Laplacian matrix and hence does not affect its spectrum. As an example of a LPS-II Ramanujan graph, we take $p=5$ and $q=41$. (It can be verified that $p, q \equiv 1 \bmod (4)$ and the Legendre symbol, $\left(\frac{p}{q}\right)=1$.) Thus, we have a non-bipartite Ramanujan graph, which is 6-regular and has 42 vertices. Fig. 1 shows the graph, thus obtained.

\section{NUMERICAL RESULTS}

This section shows how the Ramanujan graphs outperform other topologies with respect to the convergence speed of the distributed average consensus algorithm. Specifically, we fix 
the number of nodes $N$ and links $M$ and consider various topologies. We define the average degree of such graphs as, $k_{\mathrm{avg}}=\frac{2 M}{N}$ (Since the Ramanujan graphs are regular, all the nodes have the same degree $k=k_{\text {avg }}$.)

We first describe the graph topologies to be contrasted with the Ramanujan LPS-II constructions:

Regular ring lattice: This is a regular structured network in which the nodes are placed on a ring, and each node is connected to $k / 2$ nodes on either side.

Watts-Strogatz Small-World graphs [6]: It starts from a regular ring lattice with $k=k_{\mathrm{avg}}=\frac{2 M}{N}$. Then, random rewiring is conducted on all graph links. With probability $p_{w}$, a link is rewired to a different node chosen uniformly at random. Notice that the $p_{w}$ parameter controls the "randomness" of the graph in the sense that $p_{w}=0$ corresponds to the original highly structured network while $p_{w}=1$ results in a random network. Self and parallel links are prevented in the rewiring procedure and the number of links is kept constant, regardless of the value of $p_{w}$. In the sequel, we refer to the Watts-Strogatz graphs as WS-I graphs.

Erdös-Renýi random graphs: Here, we randomly choose $M$ edges out of a total of $\frac{N(N-1)}{2}$ possible edges.

Since the convergence speed is determined by the ratio $\gamma=$ $\frac{\lambda_{2}(L)}{\lambda_{N}(L)}$, see section 4 , we provide plots of the ratio $\gamma$ for the different topologies. In Fig. 2(top) we plot the ratio of $\gamma$ for the LPS-II graphs over the regular ring lattice for fixed $k=$ $k_{a v g}=18$ and varying $N$ (in the range 1000 to 2100 .) We note from the plot that the relative performance of the LPS-II graphs improves steadily with increasing $N$ (as indicated by dramatically large $\gamma_{\text {LPS-II }} / \gamma_{\text {lattice }}$ ) and in fact $\gamma_{\text {LPS-II }} / \gamma_{\text {lattice }} \approx$ 3400 for $N=2000$. Fig. 2(bottom) shows a comparison of $\gamma$ between the LPS-II graphs and the Erdös-Renýi random graphs for fixed $k_{\text {avg }}=18$ and varying $N$ (in the range 1000 to 3000.) We see that the LPS-II graphs (the top line) outperform the Erdös-Renýi graphs with respect to $\gamma$ and hence convergence speed. We also note that the LPS-II graphs give a steady value of $\gamma$ with increasing $N$, while the $\gamma$ for ErdösRenýi graphs decreases with increasing $N$. Finally Fig. 3 compares $\gamma$ of LPS-II graphs with WS-I graphs for $N=6038$ ( for different values of the rewiring probability $p_{w} \in[0,1]$ with $k_{\text {avg }}=18$.) Evidently the LPS-II graphs perform much better and we notice that in terms of $\gamma$, the LPS-II graph is about $47 \%$ better than the best WS-I graph.

\section{CONCLUSION}

In this paper we reduce the difficult combinatorial topology optimization problem for the distributed average consensus algorithm as an algebraic optimization problem, namely optimizing the ratio $\gamma$ of the graph Laplacian $L$ and the use of the Ramanujan graphs in this paper is a direct consequence. The Ramanujan graphs, specifically the LPS-II graphs, outperform the structured graphs, Erdös-Renýi random graphs
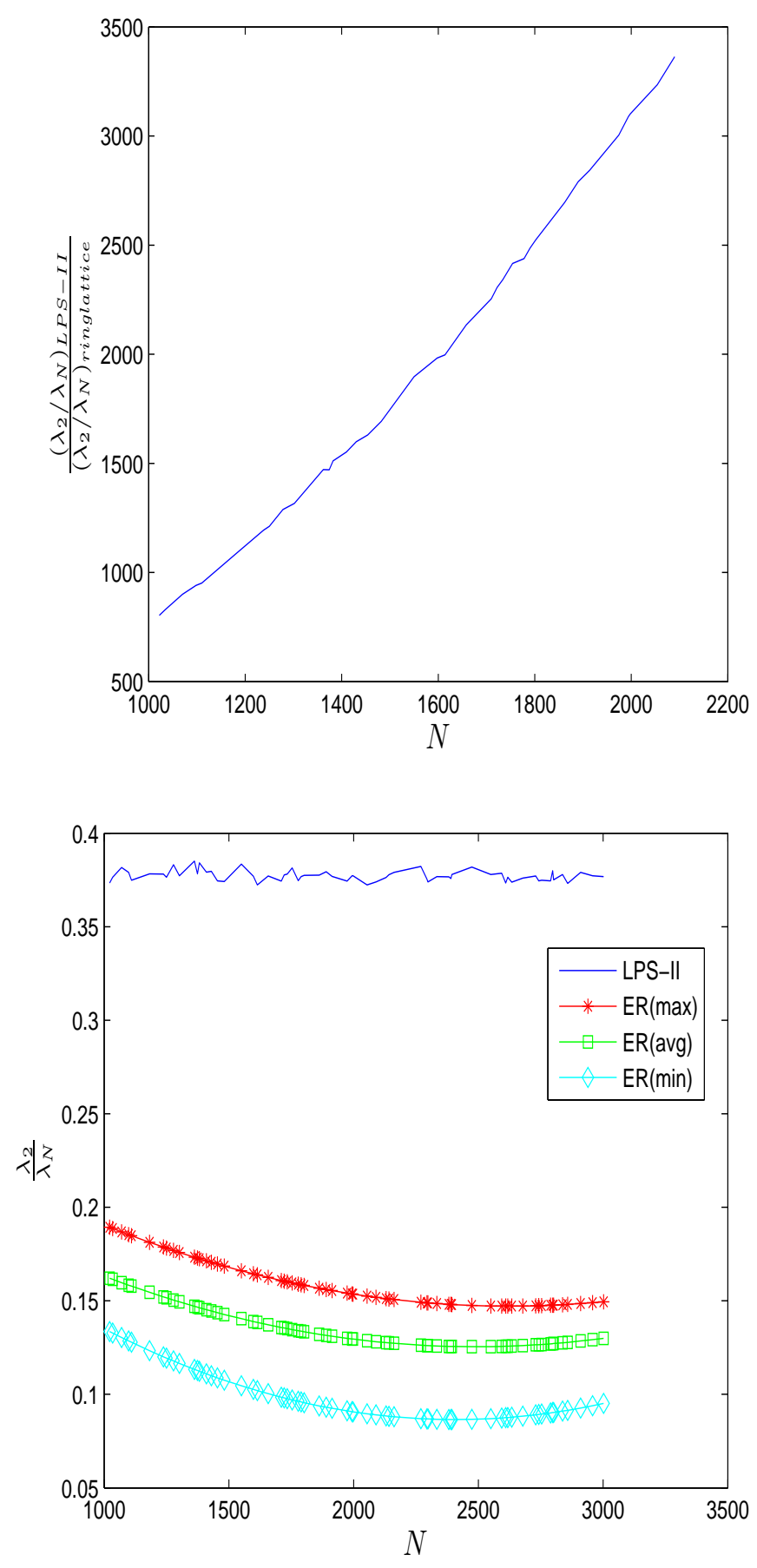

Fig. 2. Comparative study of $\gamma=\frac{\lambda_{2}(L)}{\lambda_{N}(L)}$ of different topologies. Top: LPS-II vs regular ring lattice for $k=k_{\mathrm{avg}}=18$ and varying $N$. Bottom: LPS-II vs Erdös-Renýi random graphs for fixed $k_{\text {avg }}=18$ and varying $N$. 


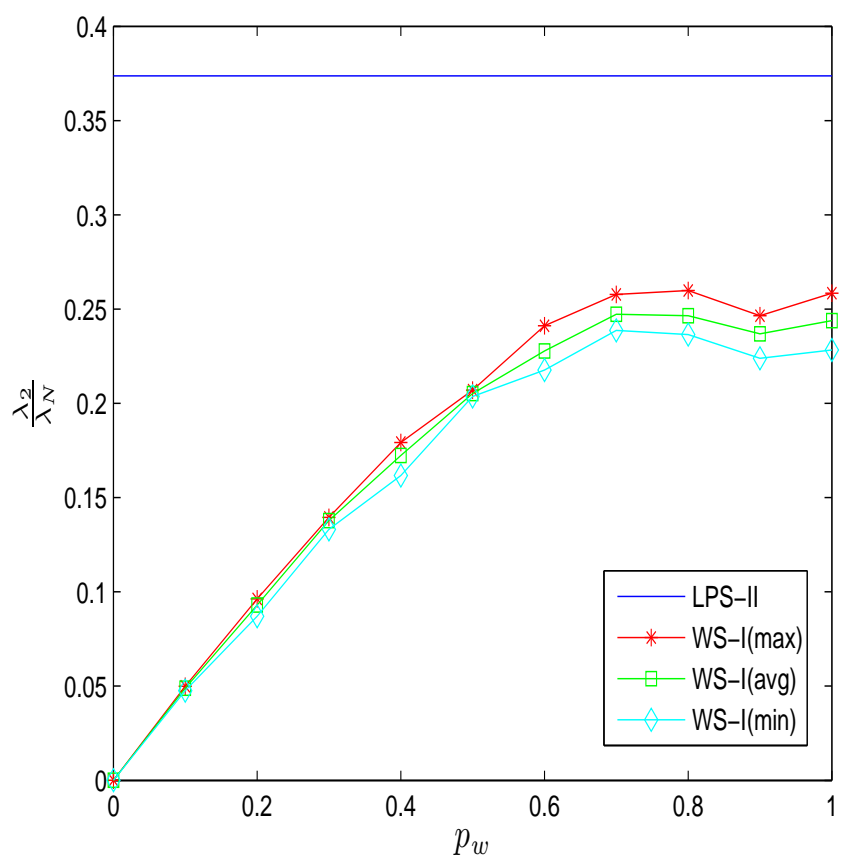

Fig. 3. Comparative study of $\gamma=\frac{\lambda_{2}(L)}{\lambda_{N}(L)}$ between LPS-II and WS-I for $N=6038$ and $k_{\text {avg }}=18$ for different values of the rewiring probability $p_{w} \in[0,1]$.

and graphs exhibiting small-world (Watts-Strogatz) property in terms of convergence speed of the distributed consensus algorithm. We also note that the relative performance of the Ramanujan graphs with respect to the other classes of graphs considered here improves steadily as the number of sensor nodes $N$ increases. In [10] we extend this approach and construct a class of random regular graphs which are close to the Ramanujan graphs for finite values of $N$.

\section{REFERENCES}

[1] R. Olfati-Saber and R. M. Murray, "Consensus problems in networks of agents with switching topology and time-delays," IEEE Trans. Automat. Contr., vol. 49, pp. 1520-1533, 2004.

[2] S. A. Aldosari and J. M. F. Moura, "Distributed detection in sensor networks: connectivity graph and smallworld networks," Asilomar Conference on Signals, Systems, and Computers, 2005.

[3] Miroslav Fiedler, "Algebraic connectivity of graphs," Czechoslovak. Mathematical Journal, vol. 23, no. 98, pp. 298-305, 1973.
[4] Fan R. K. Chung, Spectral Graph Theory, American Mathematical Society, 1997.

[5] M. Ram Murty, "Ramanujan graphs," J. Ramanujan Math. Soc, vol. 18, no. 1, pp. 1-20, 2003.

[6] D. J. Watts and S. H. Strogatz, "Collective dynamics of small-world networks," Nature, vol. 393, pp. 440-442, 1998.

[7] Jon M. Kleinberg, "Navigation in a small world," $\mathrm{Na}$ ture, vol. 406, pp. 845, Aug. 2000.

[8] Jon M. Kleinberg, "The small-world phenomenon: An algorithmic perspective," in Proc. of the thirty-second annual ACM symposium on Theory of computing, Portland, Oregon, 2000, vol. 2, pp. 63-170.

[9] A. Lubotzky, R. Phillips, and P. Sarnak, "Ramanujan graphs," Combinatorica, vol. 8, no. 3, pp. 261-277, 1988.

[10] Soummya Kar, S. A. Aldosari, and J. M. F. Moura, "Topology for distributed inference on graphs," Submitted, June 2006.

[11] L. Xiao and S. Boyd, "Fast linear iteration for distributed averaging," Syst. Contr. Lett., vol. 53, pp. 6578, Sept. 2004.

[12] Norman Biggs, Cambridge University Press, 1993.

[13] N. Alon, "Eigenvalues and expanders," Combinatorica, vol. 6, pp. 83-96, 1986.

[14] G.A. Margulis, "Explicit group-theoretical constructions of combinatorial schemes and their application to the design of expanders and concentrators," J. Probl. Inf. Transm., vol. 24, no. 1, pp. 39-46, 1988.

[15] M. Morgenstern, "Existence and explicit construction of $q+1$ regular Ramanujan graphs for every prime power q," J. Comb. Theory, ser. B, vol. 62, pp. 44-62, 1994.

[16] George Andrews, Shalsoh B. Ekhad, and Doron Zeilberger, "A short proof of Jacobi's formula for the number of representations of an integer as a sum of four squares," Amer. Math. Monthly, vol. 100, pp. 273-276, 1993.

[17] A. Berger and J. Lafferty, "Probabilistic decoding of low-density Cayley codes," Proceedings of the Canadian Workshop on Information Theory, Toronto, Canada, 1997. 